\title{
POLYNOMIAL SOLUTIONS TO CONSTANT COEFFICIENT DIFFERENTIAL EQUATIONS
}

\author{
S. PAUL SMITH
}

\begin{abstract}
Let $D_{1}, \ldots, D_{r} \in \mathbb{C}\left[\partial / \partial x_{1}, \ldots, \partial / \partial x_{n}\right]$ be constant coefficient differential operators with zero constant term. Let$$
S=\left\{f \in \mathbb{C}\left[x_{1}, \ldots, x_{n}\right] \mid D_{j}(f)=0 \text { for all } 1 \leq j \leq r\right\}
$$

be the space of polynomial solutions to the system of simultaneous differential equations $D_{j}(f)=0$. It is proved that $S$ is a module over $\mathscr{D}(V)$, the ring of differential operators on the affine scheme $V$ with coordinate ring $\mathbb{C}\left[\partial / \partial x_{1}, \ldots, \partial / \partial x_{n}\right] /\left\langle D_{1}, \ldots, D_{r}\right\rangle$. If $V$ is smooth and irreducible, then $S$ is a simple $\mathscr{D}(V)$-module, $S=1 . \mathscr{D}(V)$, and the generators for $\mathscr{D}(V)$ yield an algorithm for obtaining a basis for $S$. If $V$ is singular, then $S$ need not be simple. However, $S$ is still a simple $\mathscr{D}(V)$-module for certain curves $V$, and certain homogeneous spaces $V$, and this allows one to obtain a basis for $S$, through knowledge of $\mathscr{D}(V)$.
\end{abstract}

\section{INTRODUCTION}

Let $D_{1}, \ldots, D_{r} \in \mathbb{C}\left[\partial / \partial x_{1}, \ldots, \partial / \partial x_{n}\right]$ be constant coefficient differential operators acting on $\mathbb{C}\left[x_{1}, \ldots, x_{n}\right]$, the commutative polynomial ring in $n$ indeterminates. Our basic problem is to understand

$$
S=\left\{f \in \mathbb{C}\left[x_{1}, \ldots, x_{n}\right] \mid D_{j}(f)=0 \text { for all } 1 \leq j \leq r\right\},
$$

the space of polynomial solutions to the system of simultaneous differential equations $D_{j}(f)=0$. For example, can one give an algorithm for determining a basis for $S$ ? This paper outlines an algebraic approach to understanding $S$.

We take the following point of view. The solution space $S$ is made into a module over a certain noncommutative algebra, which we will call $R$ for now. Thus given one solution $f \in S$, acting on $f$ by elements of $R$ produces new solutions. The problem then becomes to analyse $S$ as an $R$-module. For example, if $S$ is a simple $R$-module (which it will sometimes be), then given any $0 \neq f \in S, S=R . f$; that is we can obtain all polynomial solutions by acting on $f$ by elements from $R$. To turn this idea into an effective computational tool one needs to know the elements of $R$. This is close to the heart of the problem. $R$ itself will consist of certain differential operators (with polynomial coefficients), but will be described in such a way that it is not at all easy (initially) to determine the elements of $R$. Thus our strategy for understanding $S$ falls into two parts. One is to find an effective means of determining the elements

Received by the editors December 7, 1989.

1980 Mathematics Subject Classification (1985 Revision). Primary 35E20, 16A33. 
in $R$, and the other is to understand $S$ as an $R$-module. In many cases both these questions can be adequately dealt with.

A relatively easy, but familiar, example is given by the 1-dimensional heat equation. Set $D=\partial / \partial x-\partial^{2} / \partial y^{2}$. The most important element in $R$ is $Q=2 x \partial / \partial y+y$ (see Examples $1.3(\mathrm{a})$ and 5.2). It is easy to check that $Q$ and $D$ commute with one another, and by Proposition 5.1 (which is a special case of Theorem 4.4) a basis for $S$ is given by $Q^{n}(1)(n \geq 0)$. Moreover, given $g \in \mathbb{C}[y]$, then the unique $f \in \mathbb{C}[x, y]$ such that $f(\overline{0}, y)=g(y)$ and $D(f)=0$ is given by $f=g(Q)(1)$.

Let us describe $R$. Write $\mathscr{D}=\mathbb{C}\left[x_{1}, \ldots, x_{n}, \partial / \partial x_{1}, \ldots, \partial / \partial x_{n}\right]$ for the ring of differential operators with polynomial coefficients. The natural action makes $\mathbb{C}\left[x_{1}, \ldots, x_{n}\right]$ a left $\mathscr{D}$-module. Suppose that $Q \in \mathscr{D}$ has the following property: for each $j=1, \ldots, r$ there exist $P_{j 1}, P_{j 2}, \ldots, P_{j r} \in \mathscr{D}$ such that $D_{j} Q=P_{j 1} D_{1}+\cdots+P_{j r} D_{r}$. Thus $Q$ has the property that if $f$ is a solution to the system of equations, then so too is $Q(f)$ : it is important to observe that this does not depend on $f$ being a polynomial. The set of all such $Q$ forms an associative subalgebra of $\mathscr{D}$ which contains $\mathscr{D} D_{1}+\cdots+\mathscr{D} D_{r}$ as a twosided ideal. The quotient algebra is a familiar object (to some); namely, it is anti-isomorphic to the ring of differential operators on the affine scheme, $V$ say, defined by the ideal of the commutative polynomial ring $\mathbb{C}\left[\partial / \partial x_{1}, \ldots, \partial / \partial x_{n}\right]$ generated by $D_{1}, \ldots, D_{r}$ (see $\left.\S 1\right)$. Write $\mathscr{D}(V)$ for the ring of differential operators on $V$; some basic facts about $\mathscr{D}(V)$ are given in $\S 1$. For the purposes of this discussion one may take $R$ to be either the set of all such $Q$, or the algebra $\mathscr{D}(V)$.

We shall discuss the case when $V$ is irreducible; that is, $V$ is the variety with coordinate ring $\mathbb{C}\left[\partial / \partial x_{1}, \ldots, \partial / \partial x_{n}\right] /\left\langle D_{1}, \ldots, D_{r}\right\rangle$. If one of the $D_{j}$ has a nonzero constant term (that is, $0 \notin V$ ) then 0 is the only polynomial solution (2.3), so suppose that is not the case. If $V$ is a smooth variety (in fact, if $V$ is smooth at 0 ), then $S$ is a simple module over the ring $R$, and it is easy to give an algorithm to obtain all the elements of $S$ : essentially all one needs to know are generators for the module of derivations on $V$, or equivalently the tangent vectors to $V$ at 0 . When $V$ is singular then $\mathscr{D}(V)$ is not so well understood, and consequently it is more difficult to obtain a basis for $S$ (although it is still possible to determine the dimension of the polynomials in $S$ of degree $\leq k$ ). When $V$ is of dimension 0 , then (because of the irreducibility hypothesis) $V$ is a single point, and $\operatorname{dim}_{\mathbb{C}} S=1$. Hence the first nontrivial case occurs when $V$ is a curve. In that case a lot is known about $\mathscr{D}(V)$, and consequently we obtain fairly good information about $S$. For example, one can say precisely when $S$ is a simple $\mathscr{D}(V)$-module. However, we would still like better information when $V$ is a singular curve, and there are still important questions to be answered about $\mathscr{D}(V)$ (see $\S 4)$.

When $\operatorname{dim} V \geq 2$, and $V$ is singular, one has much less information about $\mathscr{D}(V)$, and consequently less information about $S$. Thus the point of view of this paper leads to the algebraic problem of understanding $\mathscr{D}(V)$ for such $V$. Some important cases lead to singular $V$. For example, if $D=\partial / \partial x_{1}^{2}+$ $\cdots+\partial / \partial x_{n}^{2}$, then $V$ is singular. However, this is a case that can be effectively handled $(\S 6)$, in part because there is an action of the Lie algebra $\operatorname{so}(n+2)$ on $S$. In fact $S$ becomes an irreducible representation of $\operatorname{so}(n+2)$ related 
to the completely prime primitive ideal corresponding to the minimal nilpotent coadjoint orbit (the Joseph ideal). However, for general singular $V$, we are unable to obtain as strong results as we would like.

The paper is arranged as follows. $\S 1$ gives some basic information about rings of differential operators, and explains how $S$ is made into a module over the ring of differential operators, $\mathscr{D}(V) . \S \S 2$ and 3 are not part of the main theme, and are not new. They do contain some relevant material, and some of the ideas are required in later sections. $\S 2$ discusses the relationship between polynomial and $C^{\infty}$ solutions (due to Malgrange), and $\S 3$ discusses the pairing between $\mathbb{C}\left[\partial / \partial x_{1}, \ldots, \partial / \partial x_{n}\right]$ and $\mathbb{C}\left[x_{1}, \ldots, x_{n}\right]$ which allows one to determine the dimension of the space of solutions of degree $\leq k$. The main theme of the paper continues in $\S 4$ where the structure of $S$ as a $\mathscr{D}(V)$-module is described. An important result is that $S$ is simple when $V$ is smooth. $\S 4$ also discusses the difficulty of describing $\mathscr{D}(V)$ when $V$ is singular, and to what extent these difficulties can be handled when $V$ is a curve. $\S 5$ illustrates how our approach applies to an evolution equation of the form $\partial / \partial t-Q$ where $Q \in \mathbb{C}\left[\partial / \partial x_{1}, \ldots, \partial / \partial x_{n}\right] . \S 6$ gives some examples related to enveloping algebras of Lie algebras; here $V$ is always singular, but $\mathscr{D}(V)$ can be described. It is a quotient ring of the enveloping algebra of a semisimple Lie algebra, and $S$ is a simple $\mathscr{D}(V)$-module, (equivalently an irreducible representation of the Lie algebra in question). As remarked above $\partial / \partial x_{1}^{2}+\cdots+\partial / \partial x_{n}^{2}$ is one such example.

The problem of describing $S$ is both natural and basic, yet I have been unable to find any papers which deal with it. It should be emphasised that this question is purely algebraic, and can be asked over any field (even of positive characteristic); therefore there should be a purely algebraic approach to the question. This is what this paper offers. Although our techniques are algebraic, the problem itself is of wider interest. Therefore the algebraic background is given in some detail. Hopefully those to whom this material is familiar will make allowances for such pedantry.

The first observations concerning this work were made about three years ago during a visit to the University of Nijmegen. I would like to thank the mathematicians there, particularly A. van den Essen, for the hospitality, and for the stimulating environment they provided. I have had a number of very useful conversations about this material since then with T. J. Hodges, T. Levasseur and A. van den Essen. I thank them all. The ideas were first presented in a talk at Séminaire d'Algèbre Malliavin in Paris in 1985, and a brief account appears in [S1].

\section{The Ring acting on the SPaCe of solutions $S$}

This section begins with some background on rings of differential operators on algebraic varieties. A basic reference for such material is [Bj], and [SS, §1] for the case of singular varieties. Next we describe how the ring $R=\mathscr{D}(V)$ acts on $S$. Finally, an example is given to illustrate how the general principles of this paper may be applied to find all polynomial solutions of $\partial^{2} f / \partial x^{2}-$ $2 \partial f / \partial x-\partial^{2} f / \partial y^{2}=0$.

Let $V$ be an irreducible affine algebraic variety (over $\mathbb{C}$ ). We give a defintion of $\mathscr{D}(V)$ which takes advantage of the fact that $V$ is irreducible. Write $\mathscr{O}(V)$, 
or $A$, for the coordinate ring of $V$, and let $K$ be the field of rational functions on $V$. Choose a transcendence basis $t_{1}, \ldots, t_{d}$ for $K$. Each partial derivative $\partial / \partial t_{j}$ on $\mathbb{C}\left(t_{1}, \ldots, t_{d}\right)$ extends uniquely to a derivation on $K$. Define $\mathscr{D}(K)=K\left[\partial / \partial t_{1}, \ldots, \partial / \partial t_{d}\right]$; that is $\mathscr{D}(K)$ is the associative subalgebra of End $_{\mathbb{C}} K$ generated by $K$ (acting on itself by multiplication) and by the $\partial / \partial t_{j}$. Think of $\mathscr{D}(K)$ acting on $K$ from the left. Then $\mathscr{D}(A)$, or $\mathscr{D}(V)$, the ring of differential operators on $V$, is defined by

$$
\mathscr{D}(V):=\{D \in \mathscr{D}(K) \mid D(f) \in A \text { for all } f \in A\} .
$$

By construction, $A=\mathscr{O}(V)$ is a left $\mathscr{D}(V)$-module. It can be shown that $\mathscr{D}(K)$ does not depend on the choice of transcendence basis, and hence $\mathscr{D}(V)$ depends only on $V$.

Let us point out at once that while it is obvious that $A \subset \mathscr{D}(V)$, it is far from obvious how to get one's hands on the other elements of $\mathscr{D}(V)$. For $V$ smooth, this is possible. In that case $\mathscr{D}(V)=A\left[\delta_{1}, \ldots, \delta_{m}\right]$ where $\delta_{1}, \ldots, \delta_{m}$ generate $\operatorname{Der} A$, the space of $\mathbb{C}$-linear derivations on $A$, as an $A$-module. However, if $V$ is singular, it need not even be true that $\mathscr{D}(V)$ is generated by $A$ and finitely many other differential operators. This point is taken up in $\S 4$.

Given $D \in \mathscr{D}(V) \subset \mathscr{D}(K)$ define the order of $D$ in the usual way. That is, elements of $K$ are of order zero, and each $\partial_{j}$ is of order 1, etc.

Write $\mathscr{D}=\mathscr{D}\left(\mathbb{A}^{n}\right)=\mathbb{C}\left[x_{1}, \ldots, x_{n}, \partial_{1}, \ldots, \partial_{n}\right]$ for the ring of differential operators on $\mathbb{C}\left[x_{1}, \ldots, x_{n}\right]$ where $\partial_{j}=\partial / \partial x_{j}$. Thus $\mathbb{C}\left[x_{1}, \ldots, x_{n}\right]$ is a left $\mathscr{D}$-module. The ring $\mathbb{C}\left[\partial_{1}, \ldots, \partial_{n}\right]$ is a commutative polynomial ring in $n$ indeterminates. It is useful to observe that when $\mathbb{C}\left[x_{1}, \ldots, x_{n}\right]$ is considered as a $\mathbb{C}\left[\partial_{1}, \ldots, \partial_{n}\right]$-module by restriction, every nonzero submodule contains 1 . In fact, $\mathbb{C}\left[x_{1}, \ldots, x_{n}\right]$ is isomorphic to the injective envelope of the $\mathbb{C}\left[\partial_{1}, \ldots, \partial_{n}\right]$-module $\mathbb{C}\left[\partial_{1}, \ldots, \partial_{n}\right] /\left\langle\partial_{1}, \ldots, \partial_{n}\right\rangle$.

Notation. We follow the usual conventions for multi-index notation. If $\alpha=$ $\left(\alpha_{1}, \ldots, \alpha_{n}\right)$ then $x^{\alpha}=x_{1}^{\alpha_{1}} \ldots x_{n}^{\alpha_{n}}$ and $\partial^{\alpha}=\partial_{1}^{\alpha_{1}} \ldots \partial_{n}^{\alpha_{n}}$. Write $|\alpha|=\alpha_{1}+$ $\cdots+\alpha_{n}$, and $\alpha !=\left(\alpha_{1}\right) ! \cdots\left(\alpha_{n}\right) !$. If $\alpha, \beta$ are multi-indices, then $\delta_{\alpha \beta}$ is the Kronecker delta i.e., $\delta_{\alpha \beta}=1$ if $\alpha=\beta$, and 0 otherwise.

There is another useful way to describe $\mathscr{D}(V)$. Since $V$ is affine, suppose that $V \subset \mathbb{A}^{n}$. Let $I$ be the ideal of $\mathbb{C}\left[x_{1}, \ldots, x_{n}\right]$ defining $V$; that is $A=$ $\mathbb{C}\left[x_{1}, \ldots, x_{n}\right] / I$. If $D \in \mathscr{D}$ is such that $D(I) \subset I$, then there is an induced operator $\bar{D}$ on $A$. The set of all such $D$ forms a subalgebra of $\mathscr{D}, T$ say, and $\left\{D \in \mathscr{D} \mid D(f) \in I\right.$ for all $\left.f \in \mathbb{C}\left[x_{1}, \ldots, x_{n}\right]\right\}$ is a two-sided ideal of $T$; in fact this ideal is $I \mathscr{D}$. Then $\mathscr{D}(V) \cong T / I \mathscr{D}$ (for a proof see [SS, 1.6] or [MR, 15.5.13]); this can be taken as a definition of $\mathscr{D}(V)$ which applies to any affine scheme $V$. Now we give a precise description of this isomorphism. The point to be explained is this: given $D \in \mathscr{D}(V)$, how does one find an element, $F$ say, of $T \subset \mathscr{D}=\mathbb{C}\left[x_{1}, \ldots, x_{n}, \partial_{1}, \ldots, \partial_{n}\right]$ such that the image of $F$ in $\mathscr{D}(V)=T / I \mathscr{D}$ is the given $D$.

Let $\psi: \mathbb{C}\left[x_{1}, \ldots, x_{n}\right] \rightarrow \mathscr{O}(V)$ be surjective with kernel $I$. Write $y_{j}=$ $\psi\left(x_{j}\right)$. Let $D \in \mathscr{D}(V)$. We will construct $F \in \mathbb{C}\left[x_{1}, \ldots, x_{n}, \partial_{1}, \ldots, \partial_{n}\right]$ such that the action of $F$ on $\mathbb{C}\left[x_{1}, \ldots, x_{n}\right]$ satisfies $F(I) \subset I$ and $\psi\left(F\left(x^{\alpha}\right)\right)=$ $D\left(y^{\alpha}\right)$ for all multi-indices $\alpha$. Suppose that $D$ is of order $m$. For each $\alpha$ with $|\alpha| \leq m$ choose $b_{\alpha} \in \mathbb{C}\left[x_{1}, \ldots, x_{n}\right]$ such that $\psi\left(b_{\alpha}\right)=D\left(y^{\alpha}\right)$. Since $D$ is completely determined by $D\left(y^{\alpha}\right)$ with $|\alpha| \leq m$ (see [MR, 15.5.11]), it 
suffices to find an $F$ such that $F\left(x^{\alpha}\right)=b_{\alpha}$ for all $\alpha$ with $|\alpha| \leq m$. This is done recursively by defining $F_{j}$ for $j=0, \ldots, m$ and setting $F=F_{m}$. Define $F_{0}=b_{(0, \ldots, 0)}$. Given $F_{j-1}$, for each multi-index $\beta$ with $|\beta|=j$ choose $a_{\beta} \in \mathbb{C}\left[x_{1}, \ldots, x_{n}\right]$ such that

$$
b_{\beta}=F_{j-1}\left(x^{\beta}\right)+\beta ! a_{\beta} .
$$

Define

$$
F_{j}=F_{j-1}+\sum_{|\beta|=j} a_{\beta} \partial^{\beta} .
$$

By construction $F_{m}\left(x^{\alpha}\right)=b_{\alpha}$ for all $\alpha$ with $|\alpha| \leq m$. Thus $F_{m}$ is the $F$ we require. Example 1.3 below illustrates this procedure.

One more preliminary definition is required. Let $L$ be a left ideal of $\mathscr{D}$. The idealiser of $L$ is the subalgebra of $\mathscr{D}$ defined by

$$
\mathbb{I}(L):=\{D \in \mathscr{D} \mid L D \subset L\} .
$$

The ring $\mathbb{I}(L)$ contains $L$ as a two-sided ideal, and is the largest such subring of $\mathscr{D}$ (whence the terminology). It is important to note that $\mathbb{I}(L) / L \cong$ End $_{\mathscr{D}}(\mathscr{D} / L)$. There is a similar definition of the idealiser of a right ideal. One may show that $T$, appearing in the above description of $\mathscr{D}(V)$, is the idealiser of $I \mathscr{D}$ (see [MR, 15.5.9]). Thus the above says that $\mathscr{D}(V) \cong \mathbb{I}(I \mathscr{D}) / I \mathscr{D}$, and we have just described this isomorphism.

Notation. This notation is fixed throughout the paper. Let $J$ be the ideal of $\mathbb{C}\left[\partial_{1}, \ldots, \partial_{n}\right]$ generated by $D_{1}, \ldots, D_{r}$. The object of interest is

$$
S=\left\{f \in \mathbb{C}\left[x_{1}, \ldots, x_{n}\right] \mid J . f=0\right\}=\{f \in R \mid \mathscr{D} J . f=0\} .
$$

Let $V$ be the affine scheme defined by $\mathscr{O}(V)=\mathbb{C}\left[\partial_{1}, \ldots, \partial_{n}\right] / J$.

The point of view offered by this paper is encapsulated in the following two easy lemmas. They describe how to give $S$ the structure of a $\mathscr{D}(V)$ module. Thus, $S$ is no longer just a "shapeless" vector-space, but may be analysed according to its structure as a $\mathscr{D}(V)$-module.

Lemma 1.1. Let $L$ be a left ideal of $\mathscr{D}$, and set $S=\left\{f \in \mathbb{C}\left[x_{1}, \ldots, x_{n}\right] \mid L . f=\right.$ $0\}$. Then $S$ is a left module over $\mathbb{I}(L)$. Furthermore, the annihilator of $S$ in $\mathbb{I}(L)$ contains $L$. Thus $S$ is a left $\mathbb{I}(L) / L$-module.

Proof. Let $Q \in \mathbb{I}(L), D \in L$ and $f \in S$. Then $D Q \in L Q \subset L$. Therefore $D(Q(f))=D Q(f) \in L . f=0$, whence $Q(f) \in S$. The rest is obvious.

Remarks. (1) One can replace $\mathbb{C}\left[x_{1}, \ldots, x_{n}\right]$ in (1.1) by any space $\mathscr{F}$ of functions on $\mathbb{C}^{n}$ (or some subset of $\mathbb{C}^{n}$ ), provided that $\mathscr{F}$ is a $\mathscr{D}$-module.

(2) It is not at all obvious how to get one's hands on the elements in $\mathbb{I}(L)$, and unless one can do that (1.1) is of little use. Therefore the key observation (given in (1.2)) is that if $L$ is generated by constant coefficient operators then one can relate $\mathbb{I}(L)$ to an algebra which is somewhat more tractable. Of course it would be wonderful to be able to eliminate the restriction to constant coefficient operators, but this problem is infinitely more difficult.

Let $\sigma: \mathscr{D} \rightarrow \mathscr{D}$ be the anti-automorphism defined by $\sigma\left(x_{j}\right)=\partial_{j}$ and $\sigma\left(\partial_{j}\right)=x_{j}$. 
Lemma 1.2. (Notation as above). There is an anti-isomorphism $\Phi: \mathbb{I}(\mathscr{D} J) / \mathscr{D} J$ $\rightarrow \mathscr{D}(V)$. Thus, $S$ is a right $\mathscr{D}(V)$-module.

Proof. The anti-isomorphism $\sigma$ induces an anti-isomorphism $\varphi: \mathbb{I}(\mathscr{D} J) / \mathscr{D} J$ $\rightarrow \mathbb{I}(\sigma(J) \mathscr{D}) / \sigma(J) \mathscr{D}$. However, $\sigma(J)$ is an ideal of $\mathbb{C}\left[x_{1}, \ldots, x_{n}\right]$ such that $\mathscr{O}(V) \cong \mathbb{C}\left[\partial_{1}, \ldots, \partial_{n}\right] / J \cong \mathbb{C}\left[x_{1}, \ldots, x_{n}\right] / \sigma(J)$. We remarked above that, if $I$ is an ideal of $\mathbb{C}\left[x_{1}, \ldots, x_{n}\right]$, then $\mathscr{D}\left(\mathbb{C}\left[x_{1}, \ldots, x_{n}\right] / I\right) \cong \mathbb{I}(I \mathscr{D}) / I \mathscr{D}$. The result follows, with $I=\sigma(J)$.

Hence the first part of the strategy is to determine $\mathbb{I}(\mathscr{D} J)$; one does this by first determining $\mathscr{D}(V)$ (find generators), and then applying the anti-isomorphism between $\mathscr{D}(V)$ and $\mathbb{I}(\mathscr{D} J) / \mathscr{D} J$. The example shows how this works in practice.

Example 1.3. (a) Find all polynomial solutions $f \in \mathbb{C}[x, y]$ to the 1-dimensional heat equation $D(f)=\partial f / \partial x-\partial^{2} f / \partial y^{2}=0$.

The image of the operator $D$ under the anti-automorphism $\sigma$ is $x-y^{2}$, so $V$ is the curve defined by $x-y^{2}$. There is an isomorphism $\psi: \mathbb{C}[x, y] /\left\langle x-y^{2}\right\rangle \rightarrow$ $\mathbb{C}[t]$ given by $\psi(x)=t^{2}, \psi(y)=t$, hence $V$ is just the affine line $\mathbb{C}$, and $\mathscr{O}(V) \cong \mathbb{C}[t]$. Thus

$$
\mathscr{D}(V)=\mathbb{C}[t, d / d t]
$$

A preimage of $t$ in $\mathbb{C}[x, y, \partial / \partial x, \partial / \partial y]$ is $y$. To determine a preimage of $d / d t$ note that

$$
d / d t(\psi(x))=2 t \text { and } \quad d / d t(\psi(y))=1 .
$$

Hence a preimage of $d / d t$ is given by

$$
F=2 y \partial / \partial x+\partial / \partial y \text {. }
$$

Applying $\sigma$ to $F$ gives

$$
Q:=2 x \partial / \partial y+y \text {. }
$$

Therefore the point of (1.1) and (1.2) is that if $f \in S$ then so is $Q(f) \in S$. Since $1 \in S$ we have $Q^{k}(1) \in S$ for all $k$. In fact, these elements are a basis for $S$ (4.4). In particular, since $Q^{k}(1)$ is of degree $k$, there is a unique polynomial solution of degree $k$ for all $k$. It is thus routine to obtain solutions of arbitrarily high degree. The fact that $y$ is a preimage of $t$ gives the rather uninteresting fact that if $f \in S$, then so is $\sigma(y)(f)=\partial f / \partial y$. It is the operator $Q$ which is important.

(b) Find all polynomial solutions $f \in \mathbb{C}[x, y]$ to $D(f)=\partial^{2} f / \partial x^{2}-2 \partial f / \partial x$ $-\partial^{2} f / \partial y^{2}=0$.

Clearly $\sigma(D)=x^{2}-2 x-y^{2}, V$ is the curve defined by $x^{2}-2 x-y^{2}$. This curve is $\mathbb{C} \backslash\{0\}$, so $\mathscr{O}(V) \cong \mathbb{C}\left[t, t^{-1}\right]$ with $t=\psi(x-1-y), t^{-1}=$ $\psi(x-1+y)$. Thus $\mathscr{D}(V)=\mathbb{C}\left[t, t^{-1}, d / d t\right]$. Preimages of $t$ and $t^{-1}$ in $\mathbb{C}[x, y, \partial / \partial x, \partial / \partial y]$ are obvious. To determine a preimage of $d / d t$ note that

and

$$
d / d t(\psi(x))=\frac{1}{2}\left(1-t^{-2}\right)=\psi\left(y-x y-y^{2}\right)
$$

$$
d / d t(\psi(y))-\frac{1}{2}\left(-1-t^{-2}\right)=\psi\left(y-x y-y^{2}-1\right) .
$$

Hence a preimage of $d / d t$ is given by

$$
F=\left(y-x y-y^{2}\right) \partial / \partial x+\left(y-x y-y^{2}-1\right) \partial / \partial y .
$$


Applying $\sigma$ to $F$ gives

$$
Q:=(x+y)\left(\partial_{y}-\partial_{x} \partial_{y}-\partial_{y}^{2}\right)-y .
$$

Since $1 \in S$ we have $Q^{k}(1) \in S$ for all $S$. By Theorem 4.4 these elements are a basis for $S$. Again it is obvious how to obtain solutions of arbitrarily high degree. Since the preimages of the elements of $\mathscr{D}(V)$ are not unique, there is no uniqueness to the choice of operator playing the role of $Q$.

The structure of $S$ as a $\mathscr{D}(V)$-module, and the question of generators of $\mathscr{D}(V)$ is taken up in $\S 4$. $\S \S 2$ and 3 can be skipped by those wanting to continue with the main theme of the paper.

\section{Polynomial solutions versus $C^{\infty}$ solutions}

One is usually interested in more than just polynomial solutions. This section explains how the problem of $C^{\infty}$ solutions leads one to look at polynomial solutions. The explanation is the result of Malgrange [Mal] which we quote below. See [T, Chapter 1, $§ 10]$ for more details.

Notation. For each $\alpha=\left(\alpha_{1}, \ldots, \alpha_{n}\right) \in \mathbb{C}^{n}$, set $e^{\alpha, x}=e^{\left(\alpha_{1} x_{1}+\cdots+\alpha_{n} x_{n}\right)}$, write $\mathscr{P}_{\alpha}:=\mathbb{C}\left[x_{1}, \ldots, x_{n}\right] e^{\alpha, x}$ and $\mathscr{P}=\bigoplus_{\alpha} \mathscr{P}_{\alpha}$. Let $\Omega \subset \mathbb{C}^{n}$ be a nonempty open subset (in the usual topology). Write $C^{\infty}(\Omega)$ for the space of infinitely differentiable $\mathbb{C}$-valued functions on $\Omega$. Give $C^{\infty}(\Omega)$ the topology of uniform convergence. Note that $\mathscr{P} \subset C^{\infty}(\Omega)$, and that both $\mathscr{P}$ and $C^{\infty}(\Omega)$ are $\mathscr{D}$ modules.

For any space of functions $\mathscr{F}$ which is a $\mathscr{D}$-module, we write $S_{\mathscr{F}}:=\{f \in$ $\mathscr{F} \mid J . f=0\}$. In [Mal] $S_{\mathscr{P}}$ is called the space of polynomial-exponential solutions.

Theorem 2.1 (Malgrange). Let $\Omega$ be a nonempty convex open set in $\mathbb{C}^{n}$. Let $J$ be an ideal in $\mathbb{C}\left[\partial_{1}, \ldots, \partial_{n}\right]$. Then every $f \in S_{C^{\infty}(\Omega)}$ can be approximated by elements in $\bigoplus_{\alpha} S_{\mathscr{P}_{\alpha}}$.

Thus it is natural to determine the spaces $S_{\mathscr{P}_{\alpha}}=\left\{f \in \mathscr{P}_{\alpha} \mid J . f=0\right\}$. The next lemma says that determining the $S_{\mathscr{P}_{\alpha}}$ may be reduced to finding the polynomial solutions for a related system of constant coefficient differential equations.

Fix $\alpha=\left(\alpha_{1}, \ldots, \alpha_{n}\right) \in \mathbb{C}^{n}$. Let $\lambda: \mathbb{A}^{n} \rightarrow \mathbb{A}^{n}$ be the affine transformation $\lambda(q)=q-\alpha$, for $q \in \mathbb{A}^{n}$. Let $\varphi$ be the comorphism on $\mathbb{C}\left[\partial_{1}, \ldots, \partial_{n}\right]$. That is $\varphi\left(\partial_{j}\right)=\partial_{j}+\alpha_{j}$ for all $j$. Set $J^{\prime}:=\varphi(J)$, and $S^{\prime}:=\left\{f \in R \mid J^{\prime} . f=0\right\}$.

Lemma 2.2. With the above notation, $S_{\mathscr{P}_{\alpha}}=e^{-\alpha, x} S^{\prime}$.

Proof. Observe that $\left[\partial_{j}, e^{\alpha . x}\right]=\alpha_{j} e^{\alpha . x}$, whence $\left(\partial_{j}-\alpha_{j}\right)=e^{\alpha . x} \partial_{j} e^{-\alpha . x}$. Thus $\varphi(D)=e^{\alpha . x} D e^{-\alpha . x}$, and $J^{\prime}=e^{\alpha . x} J e^{-\alpha . x}$. In particular, $J^{\prime}, f=0$ if and only if $J \cdot e^{-\alpha . x} f=0$.

Sometimes $S=0$; for example, if $D=d / d x-1$, then there are no polynomial solutions, the only power series solution being $f(x)=e^{x}$. In order to have a problem to study we characterise when $S=0$.

\section{Lemma 2.3.}

(a) $S \neq 0 \Leftrightarrow 0 \in V \Leftrightarrow J \subset\left\langle\partial_{1}, \ldots, \partial_{n}\right\rangle$.

(b) $S_{\mathscr{P}_{\alpha}} \neq 0 \Leftrightarrow \alpha \in V$.

Proof. (a) If $J \subset\left\langle\partial_{1}, \ldots, \partial_{n}\right\rangle$ then $1 \in S$, and $S \neq 0$. Conversely, suppose that $J \not \subset\left\langle\partial_{1}, \ldots, \partial_{n}\right\rangle$. Let $D \in J$, with $D=Q+\mu$ where $Q \in\left\langle\partial_{1}, \ldots, \partial_{n}\right\rangle$ 
and $0 \neq \mu \in \mathbb{C}$. If $f \in \mathbb{C}\left[x_{1}, \ldots, x_{n}\right]$ then $\operatorname{deg} Q(f)<\operatorname{deg} f$, whence $\operatorname{deg} D(f)=\operatorname{deg} f$. Therefore $D(f) \neq 0$. Hence $S=0$.

(b) Follows from (a) and (2.2).

Remarks. (1) Let $p \in \mathbb{C}\left[T_{1}, \ldots, T_{n}\right]$, and set $P=p\left(\partial_{1}, \ldots, \partial_{n}\right)$. Then $P\left(e^{\alpha . x}\right)=p(\alpha) e^{\alpha . x}$, whence $e^{\alpha . x} \in S_{\mathscr{S}_{\alpha}} \Leftrightarrow \alpha \in V$. This is the key point in (2.3). Furthermore, $S_{\mathscr{P}_{\alpha}} \neq 0 \Leftrightarrow e^{\alpha . x} \in S_{\mathscr{P}_{\alpha}}$.

(2) Each $S_{\mathscr{P}_{\alpha}}$ is a right $\mathscr{D}(V)$-module, and the analysis which we will apply to $S=S_{\mathscr{P}_{0}}$ in $\S 4$ can be applied to each $S_{\mathscr{P}_{\alpha}}(\alpha \in V)$.

Hencefoth, we shall suppose that $J \subset\left\langle\partial_{1}, \ldots, \partial_{n}\right\rangle$. Write $\mathfrak{m}$ for the image of $\left\langle\partial_{1}, \ldots, \partial_{n}\right\rangle$ in $\mathscr{O}(V)=\mathbb{C}\left[\partial_{1}, \ldots, \partial_{n}\right] / J$; thus $\mathfrak{m}$ is the maximal ideal at the point $0 \in V$. We will write $A_{\mathrm{m}}$ for the local ring of $V$ at 0 .

\section{The PAIRING $\mathbb{C}\left[\partial_{1}, \ldots, \partial_{n}\right] \times \mathbb{C}\left[x_{1}, \ldots, x_{n}\right] \rightarrow \mathbb{C}$, AND THE NUMBER OF SOLUTIONS OF A GIVEN DEGREE}

Write $R_{k}=\mathbb{C}\left[x_{1}, \ldots, x_{n}\right]_{k}$ for the space of polynomials of degree $\leq k$. Set $S_{k}=S \cap R_{k}$. We show how to determine $\operatorname{dim}_{\mathbb{C}} S_{k}$, or at least how to reduce it to a problem in commutative algebra which can in some sense be solved. A trivial consequence (via the Hilbert-Samuel polynomial) is that $\operatorname{dim}_{\mathbb{C}} S_{k}$ is a polynomial in $k$ of degree $\operatorname{dim} V$ for large $k$. The problem of computing $\operatorname{dim}_{\mathbb{C}} S_{k}$ is addressed in the papers [St1] and [St2]. This approach is slightly different; for example, those papers are mainly concerned with the case where $D_{1}, \ldots, D_{r}$ are homogeneous. Some results on $\operatorname{dim}_{\mathbb{C}} S_{k}$ may also be found in [Mat].

Let $D \in \mathbb{C}\left[\partial_{1}, \ldots, \partial_{n}\right]$ and $f \in \mathbb{C}\left[x_{1}, \ldots, x_{n}\right]$. Write $\langle D, f\rangle=\left.D(f)\right|_{0}$ i.e., the evaluation of $D(f)$ at $x_{1}=\cdots=x_{n}=0$. Because $\left\langle x^{\alpha}, \partial^{\beta}\right\rangle=\delta_{\alpha \beta} \alpha$ ! this pairing is nondegenerate in the sense that the induced maps

$$
\mathbb{C}\left[\partial_{1}, \ldots, \partial_{n}\right] \rightarrow \mathbb{C}\left[x_{1}, \ldots, x_{n}\right]^{*}=\operatorname{Hom}_{\mathbb{C}}\left(\mathbb{C}\left[x_{1}, \ldots, x_{n}\right], \mathbb{C}\right)
$$

and

$$
\mathbb{C}\left[x_{1}, \ldots, x_{n}\right] \rightarrow \mathbb{C}\left[\partial_{1}, \ldots, \partial_{n}\right]^{*}=\operatorname{Hom}_{\mathbb{C}}\left(\mathbb{C}\left[\partial_{1}, \ldots, \partial_{n}\right], \mathbb{C}\right)
$$

are both injective.

Since this pairing is standard, so too are the results below. We include them for completeness, and because (3.3a) and (3.4) are required later.

Lemma 3.1. $S=\left\{f \in \mathbb{C}\left[x_{1}, \ldots, x_{n}\right] \mid\langle J, f\rangle=0\right\}$.

Proof. If $f \in S$ then certainly $\langle J, f\rangle=0$, because $P(f)=0$ for all $P \in J$. If $f \notin S$, then $P(f) \neq 0$ for some $P \in J$. Therefore, there exists $D \in$ $\mathbb{C}\left[\partial_{1}, \ldots, \partial_{n}\right]$ such that $D P(f)=1$. Hence $\langle D P, f\rangle=1 \in\langle J, f\rangle$.

Lemma 3.2.

$$
\begin{aligned}
R_{k} & =\mathbb{C}\left[x_{1}, \ldots, x_{n}\right]_{k} \\
& =\left\{f \in \mathbb{C}\left[x_{1}, \ldots, x_{n}\right] \mid\left\langle\left(\partial_{1}, \ldots, \partial_{n}\right)^{k+1}, f\right\rangle=0\right\} .
\end{aligned}
$$

Proof. Since $R_{k}$ and $\left(\partial_{1}, \ldots, \partial_{n}\right)^{k+1}$ have bases of the form $\left\{x^{\alpha}|| \alpha \mid \leq k\right\}$ and $\left\{\partial^{\beta}|| \beta \mid>k\right\}$ respectively, the result is immediate.

\section{Proposition 3.3.}

(a) $S_{k}=\left\{f \in \mathbb{C}\left[x_{1}, \ldots, x_{n}\right] \mid\left\langle\mathfrak{m}^{k+1}, f\right\rangle=0\right\} \cong\left(\mathscr{O}(V) / \mathfrak{m}^{k+1}\right)^{*}$. 
(b) $\operatorname{dim}_{\mathbb{C}} S_{k}=\operatorname{dim}_{\mathbb{C}} \mathscr{O}(V) / \mathrm{m}^{k+1}$.

(c) $\operatorname{dim} S<\infty$ if and only if $0 \in V$ is an isolated point in $\operatorname{Max} \operatorname{Spec} V$.

(d) Suppose that all the $D_{1}, \ldots, D_{r}$ are homogeneous. Then $\operatorname{dim} S<\infty$ if and only if $\operatorname{Max} \operatorname{Spec} V=\{0\}$.

(e) If $V$ is smooth at 0 , and $d=\operatorname{dim} X$, then $\operatorname{dim}_{\mathbb{C}} S_{k}=\left(\begin{array}{c}k+d \\ d\end{array}\right)$ for $k>>0$.

Proof. (a) follows from (3.1), (3.2), and the nondegeneracy of $\langle$,$\rangle . An im-$ mediate consequence is $(b)$.

Note that 0 is an isolated point of $V$ if and only if the only irreducible component of $V$ containing 0 is 0 itself. This is equivalent to $\mathfrak{m}$ being a minimal prime of $\mathscr{O}(V)$. Suppose that $\mathfrak{m}$ is not a minimal prime of $\mathscr{O}(V)$. Let $\mathfrak{p} \varsubsetneqq \mathfrak{m}$ be a prime ideal. Write $\mathfrak{n}$ for the image of $\mathfrak{m}$ in $\mathscr{O}(V) / \mathfrak{p}$. By [AM, Corollary 10.18], $\bigcap_{k=0}^{\infty} \mathfrak{n}^{k+1}=0$. Hence $\operatorname{dim} \mathscr{O}(V) / \bigcap_{k} \mathfrak{m}^{k+1}=\infty$, whence $\operatorname{dim} S=\infty$ by (b). Conversely, suppose that $\mathfrak{m}$ is a minimal prime of $\mathscr{O}(V)$. In a noetherian ring 0 is a product of the minimal prime ideals (with repetitions). Say $0=\mathfrak{p}_{1} \cdots \mathfrak{p}_{t} \mathfrak{m}^{s}$, where the $\mathfrak{p}_{j}$ are minimal primes distinct from $\mathfrak{m}$. Since $\mathfrak{m}$ is maximal, and $\mathfrak{p}_{1} \cdots \mathfrak{p}_{t} \neq 0$, it follows that $\mathscr{O}(V)=\mathfrak{m}+\mathfrak{p}_{1} \cdots \mathfrak{p}_{t}$. Hence $\mathfrak{m}^{s}=$ $\left(\mathfrak{m}+\mathfrak{p}_{1} \cdots \mathfrak{p}_{t}\right) \mathfrak{m}^{s}=\mathfrak{m}^{s+1}$. Thus $\mathscr{O}(V) / \bigcap_{k} \mathfrak{m}^{k+1}=\mathscr{O}(V) / \mathfrak{m}^{s}$ is finite dimensional. Thus $\operatorname{dim} S<\infty$. This proves (c).

Suppose that $D_{1}, \ldots, D_{r}$ are homogeneous. If $x \in V$, then $\mathbb{C} x \subset V$. Hence, if $V \neq\{0\}$, then 0 is not an isolated point, and conversely. Therefore (d) follows from (c).

Finally (e) follows from (b) and the Hilbert-Samuel polynomial [AM, Chapter 11].

Corollary 3.4. Under the natural map $S \rightarrow \operatorname{Hom}_{\mathbb{C}}(\mathscr{O}(V), \mathbb{C})=\mathscr{O}(V)^{*}$, the image of $S$ is $\left\{\vartheta \in \mathscr{O}(V)^{*} \mid \vartheta\left(\mathfrak{m}^{k}\right)=0\right.$ for $\left.k>>0\right\}$. As an $\mathscr{O}(V)$-module, this is isomorphic to the injective hull of $\mathscr{O}(V) / \mathfrak{m}$.

Proof. See [B, §1, Exercises 29-32] for the isomorphism from $\left\{\vartheta \in \mathscr{O}(V)^{*} \mid\right.$ $\vartheta\left(\mathfrak{m}^{k}\right)=0$ for $\left.k>>0\right\}$ to the injective hull of $\mathscr{O}(V) / \mathfrak{m}$.

(3.3e) has also been established by P. F. Stiller [St1, St2] when the $D_{1}, \ldots, D_{r}$ are homogeneous. He proves that the solutions of degree precisely $k$ are the global sections of a certain line bundle on the projective variety of lines on $V$. Furthermore, (3.3d) has been previously proved (for homogeneous $D_{j}$ ) by C. Micchelli [St2, Proposition 3.1].

\section{Structure of $S$ as a $\mathscr{D}(V)$-Module}

The main theme of the paper continues in this section. We describe the structure of $S$ as a $\mathscr{D}(V)$-module. It is shown that if $V$ is smooth at 0 , then $S$ is a simple $\mathscr{D}(V)$-module. The significance of $S$ being a simple $\mathscr{D}(V)$-module is that $S=1 . \mathscr{D}(V)$ because $1 \in S$. Thus (4.4) gives a precise description of a basis for $S$ in the case $V$ is smooth at 0 . A key point is that if $V$ is a smooth irreducible variety, then $\mathscr{D}(V)$ is generated by $A=\mathscr{O}(V)$ and $\operatorname{Der}_{\mathbb{C}} A$ (see [G, 16.11.2]). That is $\mathscr{D}(V)=A\left[\delta_{1}, \ldots, \delta_{m}\right]$ where $\operatorname{Der}_{\mathbb{C}} A=A \delta_{1}+\cdots+A \delta_{m}$.

Unfortunately, $S$ may be far from simple when $V$ is singular. Examples 4.9 and 4.10 are illustrations. However, there are also cases where $S$ is simple although $V$ is singular; see (4.6) and the discussion of $\partial / \partial x_{1}^{2}+\cdots+\partial / \partial x_{n}^{2}$ in $\S 6$. Hence, the problem becomes to understand $\mathscr{D}(V)$ and $S$ when $V$ is 
singular. There is no decisive information available except when $V$ is a curve. That case is treated in detail below; one can say exactly when $S$ is simple (4.6).

First, some terminology (see [SS, $\S 1$ ] for more details). As usual $A=\mathscr{O}(V)$. Let $M$ and $N$ be $A$-modules. If $a \in A$, and $\vartheta \in \operatorname{Hom}_{\mathbb{C}}(M, N)$, then we denote by $[a, \vartheta]$ the element of $\operatorname{Hom}_{\mathbb{C}}(M, N)$ defined by

$$
[a, \vartheta](m)=a \vartheta(m)-\vartheta(a m) \text { for all } m \in M \text {. }
$$

We define $\mathscr{D}_{A}(M, N)$ to be the set of all $\vartheta \in \operatorname{Hom}_{\mathbb{C}}(M, N)$ such that there exists $p \in \mathbb{N}$ with the property that

$$
\left[a_{0}\left[a_{1}\left[\cdots\left[a_{p}, \vartheta\right] \cdots\right]\right]\right]=0 \quad \text { whenever } a_{0}, a_{1}, \ldots, a_{p} \in A .
$$

We call $\mathscr{D}_{A}(M, N)$ the space of differential operators from $M$ to $N$. In general, $\mathscr{D}_{A}(M, M)$ is a ring, and in particular, it can be shown that $\mathscr{D}_{A}(A, A)=$ $\mathscr{D}(A)$. One can show that $\mathscr{D}_{A}(M, N)$ is a $\mathscr{D}_{A}(N, N)-\mathscr{D}_{A}(M, M)$ bimodule. In particular, $\mathscr{D}_{A}(A, N)$ is a right $\mathscr{D}(A)$-module for all $N$.

Consider $\mathscr{D}_{A}(A, A / \mathfrak{m})$. This is a certain subspace of $A^{*}=\operatorname{Hom}_{\mathbb{C}}(A, \mathbb{C})$. In fact, it is shown in [S1] that it consists of precisely those linear maps which vanish on some power of $\mathfrak{m}$. Hence after $(3.3 \mathrm{a}), \mathscr{D}_{A}(A, A / \mathfrak{m})$ can be identified with $S$. Moreover, all the actions of $\mathscr{D}(V)$ are compatible (see [S1, Proposition 4.1] for details). Hence the next result, which gives an extremely concrete description of $S$ as a $\mathscr{D}(V)$-module.

Proposition 4.1. As a right $\mathscr{D}(V)$-module, $S \cong \mathscr{D}(A, A / \mathfrak{m})$.

The module $\mathscr{D}(A, A / \mathfrak{m})$ also plays an important role in [DE].

The proof of (4.2) will use the fact that $\mathscr{D}(V)$ has a natural filtration, and therefore has an associated graded algebra. Once more the reader is referred to [SS, $\S 1]$ for details. The filtration is given by the order of the differential operators, and if $V$ is smooth then the associated graded ring $\operatorname{gr} \mathscr{D}(V)$ is simply the coordinate ring of the cotangent bundle to $V, T^{*} V$, which is again a smooth affine algebraic variety. The other important fact used in the proof is that if $M$ is a nonzero left $\mathscr{D}(V)$-module, then the associated graded module $\operatorname{gr} M$, which is a module over $\mathscr{O}\left(T^{*} V\right)$, has Krull dimension at least $\operatorname{dim} V$. One possible reference for this fact is $[\mathrm{Bj}]$.

Proposition 4.2. Suppose that $V$ is a smooth irreducible variety. Then

(a) $S$ is a simple $\mathscr{D}(V)$-module,

(b) $S=1 . \mathscr{D}(V)$,

(c) $S \cong \mathscr{D}(V) / \mathfrak{m} \mathscr{D}(V)$ and $\mathrm{Ann}_{\mathscr{D}}(1)=\mathfrak{m} \mathscr{D}(V)$.

Proof. By [SS, 1.3(d)], $\mathscr{D}(A, A / \mathfrak{m}) \cong \mathscr{D}(V) / \mathfrak{m} \mathscr{D}(V)$. In [SS, 1.4(g)] we claimed that $\mathfrak{m} \mathscr{D}(V)$ is a maximal ideal of $\mathscr{D}(V)$ (for $V$ smooth). The argument there was rather brief, and we take this opportunity to fill in the details. We begin by showing that $\operatorname{gr}(\mathfrak{m} \mathscr{D}(V))=\mathrm{m} \operatorname{gr} \mathscr{D}(V)$.

Note that $\operatorname{gr}(\mathfrak{m} \mathscr{D}(V)) \supset \mathfrak{m} \operatorname{gr} \mathscr{D}(V)$. Recall that $\operatorname{gr} \mathscr{D}(V) \cong S_{A}(\operatorname{Der} A)$, the symmetric algebra on $\operatorname{Der} A$, over $A=\mathscr{O}(V)$. Therefore

$$
\operatorname{gr} \mathscr{D}(V) / \mathfrak{m} \operatorname{gr} \mathscr{D}(V) \cong A / \mathfrak{m} \otimes_{A} S_{A}(\operatorname{Der} A) \cong S_{A / \mathfrak{m}}\left(A / \mathfrak{m} \otimes_{A} \operatorname{Der} A\right)
$$

where the last isomorphism depends crucially on the fact that $\operatorname{Der} A$ is a projective $A$-module. But $S_{A / \mathfrak{m}}\left(A / \mathfrak{m} \otimes_{A} \operatorname{Der} A\right)$ is a polynomial ring on $\operatorname{dim} V$ indeterminates (it should be thought of as the regular functions on the fibre of the 
cotangent bundle lying above $0 \in V)$. Therefore $\mathfrak{m g r} \mathscr{D}(V)$ is a prime ideal of $\operatorname{gr} \mathscr{D}(V)$. Hence if $\operatorname{gr}(\mathfrak{m} \mathscr{D}(V)) \neq \mathfrak{m} \operatorname{gr} \mathscr{D}(V)$, then the associated graded module of $\mathscr{D}(V) / \mathfrak{m} \mathscr{D}(V)$ has Krull dimension strictly less than $\operatorname{dim} V$. But the only such $\mathscr{D}(V)$-module is 0 . Therefore $\operatorname{gr}(\mathfrak{m} \mathscr{D}(V))=\mathfrak{m} \operatorname{gr} \mathscr{D}(V)$.

Similarly, it follows that $m \mathscr{D}(V)$ is a maximal right ideal of $\mathscr{D}(V)$ (by considering the Krull dimension of the associated graded module). The result follows from (4.1).

It is clear from some of the earlier results (for example, (2.3), (3.3) and (4.1)) that it is not so much $V$ which is relevant, but rather the structure of $V$ locally at 0 . This is made more precise by the following result.

Proposition 4.3. $S$ is also a right $\mathscr{D}\left(A_{\mathfrak{m}}\right)$-module, and the $\mathscr{D}\left(A_{\mathfrak{m}}\right)$-module action restricted to $\mathscr{D}(V) \subset \mathscr{D}\left(A_{\mathfrak{m}}\right)$ coincides with the natural $\mathscr{D}(V)$-module action. As a right $\mathscr{D}\left(A_{\mathfrak{m}}\right)$-module, $S \cong \mathscr{D}\left(A_{\mathfrak{m}}, A_{\mathfrak{m}} / \mathfrak{m} A_{\mathfrak{m}}\right) \cong \mathscr{D}\left(A_{\mathfrak{m}}\right) / \mathfrak{m} \mathscr{D}\left(A_{\mathfrak{m}}\right)$.

Proof. By (2.4), $S$ is an injective $\mathscr{O}(V)$-module, therefore divisible. Hence every $a \in \mathscr{O}(V) \backslash \mathfrak{m}$ acts as a unit on $S$. Therefore, $S \otimes_{A} A_{\mathfrak{m}}=S$. However, $S \otimes_{\mathscr{D}(A)} \mathscr{D}\left(A_{\mathfrak{m}}\right) \cong S \otimes_{\mathscr{D}(A)} \mathscr{D}(A) \otimes_{A} A_{\mathfrak{m}}=S \otimes_{A} A_{\mathfrak{m}}=S$. This gives $S$ a $\mathscr{D}\left(A_{\mathfrak{m}}\right)$-module structure.

Finally, just as $\mathscr{D}(A, A / \mathfrak{m})$ can be identified with those elements of $A^{*}$ vanishing on a power of $\mathfrak{m}$, so can $\mathscr{D}\left(A_{\mathfrak{m}}, A_{\mathfrak{m}} / \mathfrak{m} A_{\mathfrak{m}}\right)$ be identified with those elements of $A_{\mathrm{m}}^{*}$ vanishing on a power of $\mathrm{m} A_{\mathrm{m}}$. But these are the same space.

Theorem 4.4. Suppose that $V$ is smooth at 0 , and $\operatorname{dim} V=d$. Then there exist $\delta_{1}, \ldots, \delta_{d} \in \operatorname{Der} A$ such that $\operatorname{Der} A_{\mathfrak{m}}$ is free on $\delta_{1}, \ldots, \delta_{d}$. If $P_{1}, \ldots, P_{d} \in$ $\mathbb{I}(\mathscr{D} J)$ are such that $\Phi\left(P_{j}\right)=\delta_{j}(\Phi$ as in (1.2)) for $1 \leq j \leq d$, then $S$ has a $\mathbb{C}$-basis $\left\{P_{1}^{i_{1}} \ldots P_{d}^{i_{d}}(1) \mid i_{1}, \ldots, i_{d} \geq 0\right\}$.

Proof. Since $V$ is smooth at 0, Der $A_{\mathrm{m}}$ is free of rank $d$. By (4.3) $S$ may be viewed as a right $\mathscr{D}\left(A_{\mathfrak{m}}\right)$-module. As a left $A_{\mathfrak{m}}$-module $\mathscr{D}\left(A_{\mathfrak{m}}\right)$ has a basis $\delta_{1}^{i_{1}} \cdots \delta_{d}^{i_{d}}[\mathrm{G}, \S 16]$. Hence

$$
\mathscr{D}\left(A_{\mathfrak{m}}\right)=\mathfrak{m} \mathscr{D}\left(A_{\mathfrak{m}}\right)+\bigoplus \mathbb{C} \delta_{1}^{i_{1}} \cdots \delta_{d}^{i_{d}} \text {, and by }(4.2 \mathrm{c}), S=\bigoplus \mathbb{C} 1 . \delta_{1}^{i_{1}} \cdots \delta_{d}^{i_{d}}
$$

Thus (4.4) essentially solves the problem of finding a basis for $S$ when $V$ is smooth at 0 . Suppose that $V$ is smooth at all points. To find all polynomial-exponential solutions, then for each $\alpha \in V$ one must compute $S_{\mathscr{P}_{\alpha}}=e^{\alpha . x}, \mathscr{D}(V) \cong \mathscr{D}\left(A, A / \mathfrak{m}_{\alpha}\right)$ where $\mathfrak{m}_{\alpha}$ is the maximal ideal of $\mathscr{O}(V)$ at $\alpha$. Once more it is simply a matter of knowing the derivations on $\mathscr{O}(V)$, and a basis for the derivations on $A_{\mathrm{m}_{\alpha}}$.

Example. Find all polynomial-exponential solutions to

$$
D(f)=\partial^{2} f / \partial y^{2}-\partial^{3} f / \partial x^{3}+\partial f / \partial x=0 .
$$

The image of the operator $D$ under the anti-automorphism $\sigma$ is $y^{2}-x^{3}+$ $x$. Hence $V$ is the elliptic curve defined by $y^{2}-x^{3}+x$. The module of derivations $\operatorname{Der} \mathscr{O}(V)$ is free on $\delta$, where $\delta$ is the derivation on $\mathscr{O}(V)$ with preimage $\left(3 x^{2}-1\right) \partial / \partial y+2 y \partial / \partial x$ in $\mathbb{C}\left[x, y, \partial_{x}, \partial_{y}\right]$. Applying $\sigma$ gives $Q:=3 y \partial_{x}^{2}+2 x \partial_{y}-y$. Therefore $\left\{Q^{k}\left(e^{\alpha x+\beta y}\right) \mid k \geq 0,(\alpha, \beta) \in V\right\}$ is a basis for the polynomial-exponential solutions. 
We now turn to the case where $V$ is singular. If $\operatorname{dim} V \geq 2$, then $\mathscr{D}(V)$ is not well understood, so we concentrate on the case where $V$ is a curve.

After (3.3), there is a nondegenerate bilinear pairing

$$
\langle,\rangle: \mathscr{D}(A, A / \mathfrak{m}) \times A \rightarrow \mathbb{C}=A / \mathfrak{m}
$$

given by evaluation. There are two ways to think of this. By identifying $\mathscr{D}(A, A / \mathfrak{m})$ with $\mathscr{D}(V) / \mathfrak{m} \mathscr{D}(V)$, if $P \in \mathscr{D}(V)$ then $\langle\bar{P}, f\rangle=\left.P(f)\right|_{0}$. However, by identifying $\mathscr{D}(A, A / \mathfrak{m})$ with a subspace of $A^{*}$, then $\langle\bar{P}, f\rangle=P(f)$. Observe that $\mathscr{D}(A, A / \mathfrak{m})$ is a right $\mathscr{D}(V)$-module, and $A$ is a left $\mathscr{D}(V)$ module, and the pairing respects the action of $\mathscr{D}(V)$ in the sense that for all $P \in \mathscr{D}(A, A / \mathfrak{m}), f \in A, Q \in \mathscr{D}(V)$ we have $\langle P Q, f\rangle=\langle P, Q . f\rangle$.

If $N$ is a subspace of $A$, write $N^{\perp}:=\{\vartheta \in \mathscr{D}(A, A / \mathfrak{m}) \mid \vartheta(N)=0\}$. If $K$ is a subspace of $\mathscr{D}(A, A / \mathfrak{m})$, write $K^{\perp}:=\{a \in A \mid \vartheta(a)=0$ for all $\vartheta \in K\}$. It is well known that the maps $N \mapsto N^{\perp}$ and $K \mapsto K^{\perp}$ give mutually inverse maps (an order reversing isomorphism) between the lattice of ideals $I$ of $A$ such that $\operatorname{Supp}(A / I) \subset\{\mathfrak{m}\}$ (equivalently the lattice of ideals of $A_{\mathfrak{m}}$ of finite codimension), and the lattice of proper $A$-submodules of $\mathscr{D}(A, A / \mathfrak{m})$. Call these lattices $\mathscr{L}_{1}$ and $\mathscr{L}_{2}$ respectively.

Since $\langle$,$\rangle respects the \mathscr{D}(A)$-module action, the maps $N \mapsto N^{\perp}$ and $K \mapsto K^{\perp}$ restrict to mutually inverse maps between the sublattices of $\mathscr{L}_{1}$ and $\mathscr{L}_{2}$ consisting of those $A$-submodules which are actually $\mathscr{D}(A)$-submodules.

Proposition 4.5. Suppose that $V$ is an irreducible curve. Then the map $N \mapsto$ $N^{\perp}$ gives an order-reversing lattice isomorphism between the lattice of $\mathscr{D}\left(A_{\mathfrak{m}}\right)$ submodules of $A_{\mathfrak{m}}$, and the lattice of $\mathscr{D}(A)$-submodules of $\mathscr{D}(A, A / \mathfrak{m})$. In particular, if $A_{\mathfrak{m}}$ is a simple $\mathscr{D}\left(A_{\mathfrak{m}}\right)$-module, then $S$ is a simple $\mathscr{D}(V)$-module. This is the case if $\mathscr{D}(V)$ is a simple ring.

Proof. Since $V$ is a curve, every nonzero ideal of $A$ is automatically of finite codimension, so the sublattice of $\mathscr{L}_{1}$ consisting of the $\mathscr{D}(A)$-submodules is in fact all the $\mathscr{D}(A)$-submodules $I$ with $\operatorname{Supp}(A / I) \subset\{\mathfrak{m}\}$. This is the same as the lattice of $\mathscr{D}\left(A_{\mathfrak{m}}\right)$-submodules of $A_{\mathfrak{m}}$. The result follows.

Theorem 4.6. Let $V$ be an irreducible curve. Then $S$ is a simple $\mathscr{D}(V)$-module if and only if $V$ has only one branch at 0 .

Proof. By [SS, 4.2] $\mathscr{O}(V)$ is a simple $\mathscr{D}(V)$-module if and only if the normalisation map $\pi: \widetilde{V} \rightarrow V$ is injective. The local version of this is that $A_{\mathfrak{m}}$ is a simple $\mathscr{D}\left(A_{\mathfrak{m}}\right)$-module if and only if $V$ has only one branch at 0 . Now apply (4.5).

Also see [DE, $\S 3 ; \mathrm{S} 1, \S 4]$ for proofs of (4.6).

Proposition 4.7. Let $V$ be an irreducible curve. Then $S$ is a finite length $\mathscr{D}(V)$ module.

Proof. This follows from $(4.5)$, because $\mathscr{O}(V)$ is of finite length as a $\mathscr{D}(V)$ module [SS].

Example 4.8. This shows how to use (4.4).

Consider the eigenvalue problem $\Delta f=\lambda^{2} f(\lambda \in \mathbb{C})$ for the Laplacian $\Delta=$ $\partial^{2} / \partial x^{2}+\partial^{2} / \partial y^{2}+\partial^{2} / \partial z^{2}$. Thus we consider the single operator $D=\Delta-\lambda^{2}$, and we show how to obtain all polynomial-exponential solutions. 
First suppose that $\lambda \neq 0$. Then $V$ is the complex 2-sphere $x^{2}+y^{2}+z^{2}-\lambda^{2}$ which is smooth, and $\operatorname{Der} \mathscr{O}(V)$ is generated by $x \partial_{y}-y \partial_{x}, y \partial_{z}-z \partial_{y}, z \partial_{x}-$ $x \partial_{z}$. Let $(\alpha, \beta, \gamma) \in V$. Then any two of these derivations are free generators of Der $A_{(\alpha, \beta, \gamma)}$ where $A_{(\alpha, \beta, \gamma)}$ is the local ring at $(\alpha, \beta, \gamma)$. Applying the antiisomorphism $\sigma$ to these elements just fixes them. Now $e^{\alpha x+\beta y+\gamma z}$ is a solution, and the space $S_{\mathscr{P}_{(\alpha, \beta, \gamma)}}$ has $\mathbb{C}$-basis $\left(x \partial_{y}-y \partial_{x}\right)^{i}\left(y \partial_{z}-z \partial_{y}\right)^{j}\left(e^{\alpha x+\beta y+\gamma z}\right)$.

Now suppose that $\lambda=0$, and let $(\alpha, \beta, \gamma) \in V$. If $(\alpha, \beta, \gamma) \neq(0,0,0)$ then this is a smooth point, and so can be treated as above. The point $(0,0,0)$ is singular, so it is not immediately clear whether or not $S$ is a simple $\mathscr{D}(V)$ module. Certainly applying any of the above derivations to $1 \in S$ is of no use at all. Nevertheless it turns out that $S$ is a simple $\mathscr{D}(V)$-module, and this example is a special case of those covered in $\S 6$.

Example 4.9. This is an example where $S$ is of infinite length as a $\mathscr{D}(V)$ module.

Consider $D=\partial_{x}^{3}+\partial_{y}^{3}+\partial_{z}^{3}$. As [BGG] shows, the ring of differential operators on the cubic cone $V$, given by $x^{3}+y^{3}+z^{3}=0$, is badly behaved. For example, $\mathscr{D}(V)$ has an infinite descending chain of two-sided ideals. Here we show that there is an infinite ascending chain of $\mathscr{D}(V)$-submodules of $S$.

Claim. If $\mathfrak{m}$ is the maximal ideal of $\mathscr{O}(V)$ corresponding to the singular point at 0 , then $\mathfrak{m}^{k}$ is a $\mathscr{D}(V)$-submodule for all $k \geq 0$.

Proof. For $i \geq 0$, set $E^{i}:=$ the homogeneous elements of degree $i$ in $\mathscr{O}(V)$. Note that $\mathfrak{m}^{k}=\bigoplus_{i \geq k} E^{i}$. For $j \in \mathbb{Z}$, set $j \mathscr{D}(V)=\left\{\vartheta \in \mathscr{D}(V) \mid \vartheta\left(E^{n}\right) \subset\right.$ $E^{n+j}$ for all $\left.n\right\}$. For each $m \geq 0$, set $I_{k}:=\bigoplus_{j \geq k}^{j} \mathscr{D}(V)$. One of the main results of $[\mathrm{BGG}]$ is that each $I_{k}$ is a two-sided ideal of $\mathscr{D}(V)$. Hence $I_{k} \cdot \mathscr{O}(V)$ is a $\mathscr{D}(V)$-submodule of $\mathscr{O}(V)$. But $I_{k} \cdot \mathscr{O}(V)=\mathrm{m}^{k}$, and therefore $\mathscr{O}(V) \supset \mathfrak{m} \supset \mathfrak{m}^{2} \supset \cdots$ is an infinite descending sequence of $\mathscr{D}(V)$-submodules of $\mathscr{O}(V)$.

By (4.5), there is therefore an infinite ascending chain of $\mathscr{D}(V)$-submodules of $S$, namely $0 \subset \mathfrak{m}^{\perp} \subset\left(\mathfrak{m}^{2}\right)^{\perp} \subset \cdots$.

Example 4.10. This is the easiest case where $V$ is a curve, and $S$ is not a simple module.

Let $D=\partial_{y}^{2}-\partial_{x}^{3}-\partial_{x}^{2}$. Thus $V$ is the plane curve defined by $y^{2}=x^{2}(x+1)$. This is a rational curve, with parametrisation $x=t^{2}-1$, and $y=t\left(t^{2}-1\right)$. There are two branches at 0 , so by (4.6) $S$ is not a simple $\mathscr{D}(V)$-module. In [SS, 4.4b] it is shown that

$$
\mathscr{D}(V)=\mathbb{C} \oplus\left(t^{2}-1\right) \mathbb{C}[t, d / d t] \subset \mathbb{C}[t, d / d t] .
$$

The only proper two-sided ideal of $\mathscr{D}(V)$ is $\left(t^{2}-1\right) \mathbb{C}[t, d / d t]$, and the only proper $\mathscr{D}(V)$-submodule of $\mathscr{O}(V)$ is $\mathfrak{m}=\left(t^{2}-1\right)=(x, y)$.

By (4.5), $S$ has a unique proper $\mathscr{D}(V)$-submodule, namely $\mathbb{C}$, and $S / \mathbb{C}$ is a simple $\mathscr{D}(V)$-module. Thus to determine $S$, a key point is to find an element of $S \backslash \mathbb{C}$. One can see at once that such elements include $x, y, x y, x^{2}+y^{2}$. Since $\operatorname{dim} \mathfrak{m}^{k} / \mathfrak{m}^{k+1}=2$ for $k \geq 1$, it follows from (3.3) that a basis for the space of solutions of degree $\leq 2$ is given by $\left\{1, x, y, x y, x^{2}+y^{2}\right\}$. Finding solutions of degree $\geq 2$ is not quite so trivial.

Consider the elements $\left(t^{2}-1\right) \partial$ and $\left(t^{2}-1\right) \partial^{2}$ of $\mathscr{D}(V)$. Some (nasty) calculations show that elements of $\mathbb{I}(\mathscr{D} D)$ mapping to these are the operators 
$P=2 x \partial_{y}+3 y \partial_{x}^{2}+2 y \partial_{x}$ and

$Q=\left(4 x^{2}+3 y^{2}\right) \partial_{x}^{2}+12 x y \partial_{x} \partial_{y}+9 y^{2} \partial_{y}^{2}+\left(4 x^{2}+2 x+4 y^{2}\right) \partial_{x}+(8 x y+6 y) \partial_{y}$.

Notice that $Q$ raises degree but $P$ does not. In particular, one finds that

$Q(x y)=20 x y+12 x^{2} y+4 y^{3}$ and $Q\left(x^{2}+y^{2}\right)=8 x^{3}+24 x y^{2}+12 x^{2}+36 y^{2}$.

Hence a basis for the solutions of degree $\leq 3$ is

$$
\left\{1, x, y, x y, x^{2}+y^{2}\right\} \cup\left\{3 x^{2} y+y^{3}, x^{3}+3 x y^{2}+3 y^{2}\right\} \text {. }
$$

Thus to obtain elements spanning $S$ it suffices to continue acting on these solutions by $P$ and $Q$.

Example 4.11. There are some interesting examples of curves $V$ where (4.6) applies. That is, $V$ is singular at 0 , but $S$ is a simple $\mathscr{D}(V)$-module. The easiest such example is when $V$ is defined by $y^{2}-x^{3}$, corresponding to $\partial^{2} f / \partial y^{2}-$ $\partial^{3} f / \partial x^{3}=0$.

Let $\Lambda \subset \mathbb{N}$ be a submonoid (subsemigroup) such that $\mathbb{N} \backslash \Lambda$ is finite. Let $V \subset \mathbb{A}^{n}$ be the curve with the property that $\mathscr{O}(V)=\mathbb{C} \Lambda$, i.e., $\mathbb{C}[t] \supset \mathscr{O}(V)=$ $\bigoplus_{\lambda \in \Lambda} \mathbb{C} t^{\lambda}$. Set $\partial=d / d t$. For each $k \in \mathbb{Z}$ set $\Lambda_{k}=\Lambda \backslash(\Lambda-k)$. Then $\mathscr{D}(V)$ contains $\mathbb{C}[t \partial]$, and is in fact a free $\mathbb{C}[t \partial]$-module with basis $t^{k} f_{k}$ where $f_{k}=\prod_{j \in \Lambda_{k}}(t \partial-j)$. Given the description of $S$ as those elements in $A^{*}$ vanishing on some power of $\mathfrak{m}$, and the pairing described above, it follows that a basis for $S$ is given by $\left\{1 . t^{-i} f_{-i} \mid i \in \Lambda\right\}$. Now it is shown in [P, 4.2] that the elements $t^{-i} f_{-i}$ span a subalgebra of $\mathscr{D}(V)$ which is isomorphic to $\mathscr{O}(V)$; the isomorphism is given by $t^{-i} f_{-i} \mapsto t^{i}$. Hence $S$ is a free module over this subalgebra of $\mathscr{D}(V)$.

In order to describe $S$ proceed as follows. Let $0 \neq \lambda$ be the smallest element of $\Lambda$. There is a finite set $\Gamma$ such that $\Lambda$ is the disjoint union of the sets

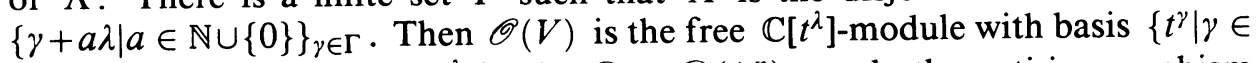
$\Gamma\}$. Find a preimage of $t^{-\lambda} f_{-\lambda}$ in $\mathscr{D}=\mathscr{D}\left(\mathbb{A}^{n}\right)$, apply the anti-isomorphism $\sigma$ to obtain $P_{\lambda}$ say. Do the same for each $\gamma \in \Gamma$ to obtain $P_{\gamma}$. Then a basis for $S$ is given by $\left\{P_{\gamma} P_{\lambda}^{k}(1) \mid \gamma \in \Gamma, k \geq 0\right\}$.

\section{Evolution EQUATIONS}

Introduce another variable, $t$ say, which may be thought of as time, and think of the $x$ 's as space coordinates. Accordingly set

$$
\mathscr{D}=\mathbb{C}\left[t, x_{1}, \ldots, x_{n}, \partial_{t}, \partial_{1}, \ldots, \partial_{n}\right]
$$

where $\partial_{t}=\partial / \partial t$. Let $D=\partial_{t}-Q$ where $Q \in \mathbb{C}\left[\partial_{1}, \ldots, \partial_{n}\right]$. A differential equation, $D(f)=0$ with $D$ of this form is called an evolution equation. A realistic physical example is the heat equation, which we discuss below.

Such an evolution equation is nicely handled by our technique. First there is an explicit description of the $P_{j}$ occurring in (4.4), and secondly it is easy to obtain a solution to an associated "initial value problem" when the initial value is a polynomial. Recall that $[Q, x]$ denotes $Q x-x Q$.

Proposition 5.1. Let $D=\partial_{t}-Q$ where $Q \in \mathbb{C}\left[\partial_{1}, \ldots, \partial_{n}\right]$. Set $S=\{f \in$ $\left.\mathbb{C}\left[t, x_{1}, \ldots, x_{n}\right] \mid D(f)=0\right\}$. For $1 \leq j \leq n$, set $P_{j}=x_{j}+t\left[Q, x_{j}\right]$. Then 
(a) the $P_{j}$ commute with one another and $\mathbb{C}\left[P_{1}, \ldots, P_{n}\right]$ is an n-dimensional polynomial ring,

(b) $S=\mathbb{C}\left[P_{1}, \ldots, P_{n}\right] .1$ and $S$ is a free $\mathbb{C}\left[P_{1}, \ldots, P_{n}\right]$-module;

(c) given $g \in \mathbb{C}\left[x_{1}, \ldots, x_{n}\right]$, there is a unique $f \in \mathbb{C}\left[t, x_{1}, \ldots, x_{n}\right]$ such that $D(f)=0$ and $f\left(0, x_{1}, \ldots, x_{n}\right)=g\left(x_{1}, \ldots, x_{n}\right)$; moreover

$$
f=g\left(P_{1}, \ldots, P_{n}\right)(1) \text {; }
$$

(d) all the $P_{j}$ commute with $D$.

Proof. Clearly $\mathbb{C}\left[\partial_{t}, \partial_{1}, \ldots, \partial_{n}\right] /\langle D\rangle \cong \mathbb{C}\left[\partial_{1}, \ldots, \partial_{n}\right]$ so $V \cong \mathbb{A}^{n}$. In particular, $V$ is smooth and irreducible, so $S$ is a simple $\mathscr{D}(V)$-module by (4.2). Write $\mathscr{O}(V)=\mathbb{C}\left[y_{1}, \ldots, y_{n}\right]$, and $\mathscr{D}(V)=\mathbb{C}\left[y_{1}, \ldots, y_{n}, \partial / \partial y_{1}, \ldots, \partial / \partial y_{n}\right]$. By (4.4), $S=1 . \mathbb{C}\left[\partial / \partial y_{1}, \ldots, \partial / \partial y_{n}\right]$, and is a free module over the commutative polynomial ring $\mathbb{C}\left[\partial / \partial y_{1}, \ldots, \partial / \partial y_{n}\right]$.

As in $\S 1$, let $\psi: \mathbb{C}\left[t, x_{1}, \ldots, x_{n}\right] \rightarrow \mathscr{O}(V)$ be given by $\psi\left(x_{j}\right)=y_{j}$ and $\psi(t)=Q\left(y_{1}, \ldots, y_{n}\right)$. To find a preimage in $\mathscr{D}$ of $\partial / \partial y_{j}$ consider the action of $\partial / \partial y_{j}$ on $\psi(t), \psi\left(x_{1}\right), \ldots, \psi\left(x_{n}\right)$. Choose $b_{j} \in \mathbb{C}\left[t, x_{1}, \ldots, x_{n}\right]$ such that $\psi\left(b_{j}\right)=\partial / \partial y_{j}(\psi(t))$. Then a preimage of $\partial / \partial y_{j}$ is $b_{j} \partial_{t}+\partial_{j}$. Take $P_{j}=\sigma\left(b_{j} \partial_{t}+\partial_{j}\right)$ and apply (4.4). There is some choice in $b_{j}$, but one such choice leads to the $P_{j}$ defined in the statement of (5.1). A calculation shows that the elements $P_{j}$ are pairwise commuting.

Thus by (4.4), $S=\mathbb{C}\left[P_{1}, \ldots, P_{n}\right] .1$. This proves (a) and (b).

(c) Set $B=\mathbb{C}\left[t, x_{1}, \ldots, x_{n}\right]$. The problem is to find $f \in S \cap(g+t B)$. However, it follows from the form of the $P_{j}$ that $g\left(P_{1}, \ldots, P_{n}\right)=g\left(x_{1}, \ldots, x_{n}\right)+P$ for some $P \in t \mathscr{D}$. Therefore $\left.g\left(P_{1}, \ldots, P_{n}\right)(1)\right|_{t=0}=g\left(x_{1}, \ldots, x_{n}\right)$. Thus $f=g\left(P_{1}, \ldots, P_{n}\right)(1)$ is a solution to the initial value problem.

To see the uniqueness, suppose that $f_{1}$ and $f_{2}$ are both solutions to the initial value problem. Then $f_{1}-f_{2} \in t B$, and $D\left(f_{1}-f_{2}\right)=0$. Suppose that $f_{1} \neq f_{2}$. Then $f_{1}-f_{2}=t^{k} h$ for some $h \in B \backslash t B$, and $0=D\left(t^{k} h\right)=$ $k t^{k-1} h+t^{k} \partial h / \partial t+t^{k} Q(h)$. Whence $h \in t B$, contradicting the choice of $h$. So, we must have $f_{1}=f_{2}$.

Similarly, the polynomial-exponential solutions are given by

$$
\mathbb{C}\left[P_{1}, \ldots, P_{n}\right] . e^{\alpha_{1} x_{1}+\cdots \alpha_{n} x_{n}-Q\left(\alpha_{1}, \ldots, \alpha_{n}\right) t}
$$

where $\left(\alpha_{1}, \ldots, \alpha_{n}\right) \in \mathbb{C}^{n}$.

Example 5.2. (a) Consider the (3-dimensional) heat equation viz. $D=\partial_{t}-$ $\partial_{x}^{2}-\partial_{y}^{2}-\partial_{z}^{2}$. By (5.1), $S=\mathbb{C}\left[x+2 t \partial_{x}, y+2 t \partial_{y}, z+2 t \partial_{z}\right] .1$. If we label these three operators as $P_{x}, P_{y}$ and $P_{z}$ respectively, then $\left\{P_{x}^{j} P_{y}^{j} P_{z}^{k}(1) \mid i, j, k \geq 0\right\}$ is a basis for $S$.

(b) Consider the 1-dimensional heat equation. Write $P=x+2 t \partial_{x}$. We show how $P$ interacts with some of the standard features of the heat equation (see [C and W]). By (5.1) $\left\{P^{n}(1) \mid n \geq 0\right\}$ is a basis for the space of polynomial solutions to the heat equation. The "heat polynomials" $v_{n}(x, t)$ are defined via the generating function

$$
e^{x z+t z^{2}}=\sum_{n=0}^{\infty} v_{n}(x, t) z^{n} / n !
$$

Since $e^{x z+t z^{2}}$ is a solution to the heat equation (because $\left(z^{2}, z\right) \in V$; $\left.\mathrm{cf} . \S 2\right)$, so too is each $v_{n}(x, t)$. What is interesting from our point of view is that 
$v_{n}(x, t)=P^{n}(1)$; this is routine to check. There is an explicit formula for $v_{n}(x, t)$ which is obtained by writing $e^{x z+t z^{2}}=e^{x z} e^{t z^{2}}$ and multiplying out the power series. As we will now explain, our point of view gives an alternative way to obtain this explicit description. First observe that there is an automorphism, $\vartheta$ say, of $\mathscr{D}$ given by $x \mapsto x+2 t \partial_{x}=P, \partial_{t} \mapsto \partial_{t}-\partial_{x}^{2}, \partial_{x} \mapsto \partial_{x}, t \mapsto t$. Let $\delta$ be the inner derivation of $\mathscr{D}$ given by $\delta=\operatorname{ad}\left(t \partial_{x}^{2}\right)$; that is $\delta(r)=t \partial_{x}^{2} r-r t \partial_{x}^{2}$. Then $\delta$ is locally nilpotent on $\mathscr{D}$, so $\exp (\delta)$ is an automorphism of $\mathscr{D}$. The point is that $\vartheta=\exp (\delta)$. Now $P^{n}=\vartheta\left(x^{n}\right)$, so

$$
\begin{aligned}
P^{n}(1) & =\sum_{k=0}^{\infty}(1 / k !)\left[\operatorname{ad}\left(t \partial_{x}^{2}\right)\right]^{k}\left(x^{n}\right)(1) \\
& =\sum_{k=0}^{\infty}(1 / k !)\left[t \partial_{x}^{2}\right]^{k}\left(x^{n}\right) \quad \text { because }\left(t \partial_{x}^{2}\right)(1)=0 \\
& =\sum_{k=0}^{\infty}\left(t^{k} / k !\right) \partial_{x}^{2^{k}}\left(x^{n}\right) \\
& =n ! \sum_{k=0}^{\infty}\left(t^{k} / k !\right) x^{n-2 k} /(n-2 k) !
\end{aligned}
$$

which is the usual expression for $v_{n}(x, t)$.

The fundamental solution to the 1-dimensional heat equation is

$$
K(x, t)=(4 \pi t)^{1 / 2} \exp \left[-x^{2} / 4 t\right] .
$$

Note that $P(K(x, t))=0$. The solution to the initial value problem $f(x, 0)=$ $g(x)$ is

$$
f(t, x)=\int_{\mathbb{C}}(4 \pi t)^{1 / 2} \exp \left[-(x-y)^{2} / 4 t\right] g(y) d y .
$$

By (5.1), if $g$ is a polynomial then $f(x, t)=g(P)(1)$. Arguably, using $P$ is a simpler way to obtain the solution.

This simple example illustrates the ideas involved in this paper; the same technique applies to other evolution equations. Both [C] and [W] have a section on how to obtain new solutions from known solutions, but neither book mentions the role played by $P$. I do not know whether the operator $P$ has been "discovered" before.

\section{SPHERICAL HARMONICS AND OTHER EXAMPLES RELATED TO ENVELOPING ALGEBRAS OF LIE ALGEBRAS}

Let $n \geq 3$, and set $D=\partial_{1}^{2}+\cdots+\partial_{n}^{2}$. Then $V$ is the quadratic cone $x_{1}^{2}+\cdots+x_{n}^{2}=0$ in $\mathbb{C}^{n}$. This has a singularity at 0 , so $\mathscr{D}(V)$ is difficult to understand. Write $U(\operatorname{so}(n+2))$ for the enveloping algebra of the Lie algebra so $(n+2, \mathbb{C})$. In [LSS] it is shown that there is a surjective map

$$
\varphi: U(\operatorname{so}(n+2)) \rightarrow \mathscr{D}(V)
$$

such that the kernel is the Joseph ideal of $U(\operatorname{so}(n+2))$ (see also [L]). The Joseph ideal is a distinguished primitive (indeed maximal) ideal of $U(\operatorname{so}(n+2)$ ) (see [J]). In particular, $\mathscr{D}(V)$ is a simple ring (whence $S$ is a simple $\mathscr{D}(V)$-module), and $\mathscr{D}(V)$ is finitely generated over $\mathbb{C}$. 
The following basis for $\varphi(\operatorname{so}(n+2))$ is explicitly given in [L]:

$$
\begin{array}{ll}
\sum_{i=1}^{n} x_{i} \partial_{i}+(n-2) / 2 ; & \\
x_{j}\left(\frac{1}{2} \sum_{i=1}^{n} \partial_{i}^{2}\right)-\left(\sum_{i=1}^{n} x_{i} \partial_{i}+(n-2) / 2\right) \partial_{j}, & 1 \leq j \leq n, \\
x_{j}, & 1 \leq j \leq n, \\
x_{k} \partial_{m}-x_{m} \partial_{k} & 1 \leq k<m \leq n .
\end{array}
$$

Applying the anti-automorphism $\sigma$, gives the following elements of $\mathbb{I}(\mathscr{D} D)$ :

$$
\begin{array}{ll}
\sum_{i=1}^{n} x_{i} \partial_{i}+(n-2) / 2, & \\
P_{j}:=\left(\frac{1}{2} \sum_{i=1}^{n} x_{i}^{2}\right) \partial_{j}-x_{j}\left(\sum_{i=1}^{n} x_{i} \partial_{i}+(n-2) / 2\right), & 1 \leq j \leq n, \\
\partial_{j}, & 1 \leq j \leq n, \\
x_{k} \partial_{m}-x_{m} \partial_{k}, & 1 \leq k<m \leq n .
\end{array}
$$

In [L] it is shown that the $P_{j}$ commute with one another. Using the PoincaréBirkhoff-Witt Theorem, it is easy to see that $\mathbb{I}(\mathscr{D} D) .1=\mathbb{C}\left[P_{1}, \ldots, P_{n}\right] .1$.

It is clear that $D$ possesses an $\mathrm{SO}(n)$ symmetry, and hence there will be an action of $\operatorname{so}(n)$ on the space $S$. However, the fact that there is an $\operatorname{so}(n+2)$ action on $S$ is much more of a surprise, and is revealing a "hidden symmetry".

There are a number of other related examples where the system of differential equations has a large degree of symmetry, and $S$ can be effectively computed although $V$ is singular at 0 . It is more efficient to describe $V$ than the system of differential equations. Let $V$ be one of the following varieties:

(A) all $p \times q$ matrices of rank $\leq k(1 \leq k<q \leq p)$,

(B) all symmetric $n \times n$ matrices of rank $\leq k(1 \leq k<n)$,

(C) all skew-symmetric $n \times n$ matrices of rank $\leq 2 k(2 \leq 2 k<n-1)$.

Each $V$ has a singularity at 0 . However, it is proved in [LS] that $\mathscr{D}(V)$ is a finitely generated simple ring. Therefore $S$ is a simple $\mathscr{D}(V)$-module. In each case $\mathscr{D}(V)$ is a factor ring of the enveloping algebra of a semisimple Lie algebra: these are $\mathrm{gl}(p+q), \operatorname{sp}(2 n), \operatorname{so}(2 n)$ in cases $A, B$, and $C$ respectively. Explicit generators for $\mathscr{D}(V)$ are given in [LS, Chapter IV, $\S 1.9]$ (although the expressions there do not make it easy to compute the appropriate preimages in $\mathscr{D})$. I do not know if these systems of differential equations arise anywhere, nor if there is any other way to describe all the polynomial solutions.

The easiest case is $A$ with $k=1, p=q=2$. Then there is a single differential equation in variables $x_{11}, x_{12}, x_{21}, x_{22}$; the operator is $\partial_{11} \partial_{22}-$ $\partial_{12} \partial_{21}$. Using [LS] gives $S=\mathbb{C}\left[P_{11}, P_{12}, P_{21}, P_{22}\right] .1$ where

$$
P_{i j}=x_{i j}+\sum_{1 \leq a, b \leq 2} x_{i a} x_{b j} \partial_{b a} .
$$

Questions. The issues raised in this paper lead to the following questions.

1. When is $\mathscr{D}(V)$ a finitely generated algebra? When is $\mathscr{D}(V)$ a simple ring? When is $\mathscr{O}(V)$ a simple module? When is $S$ a simple module? 
2. If $\mathscr{D}(V)$ is finitely generated, give a procedure for obtaining a set of generators. For example, find an integer $k$, such that $\mathscr{D}(V)$ is generated by those operators of order $\leq k$.

3 . Let $M$ be a cyclic $\mathscr{D}$-module. Study End $_{\mathscr{D}} M$. When is it finitely generated etc.? If the ideas of this paper are to be extended to differential operators with nonconstant (polynomial) coefficients, then one must first understand the rings End $\mathscr{D} M$. A starting point might be the following question.

4. Let $J$ be an ideal of $\mathbb{C}\left[x_{1}, \ldots, x_{n}\right]$ defining a smooth variety. What properties of the module $\mathscr{D} / J \mathscr{D}$ lead to the ring $\operatorname{End}_{\mathscr{D}}(\mathscr{D} / J \mathscr{D})$ being a finitely generated, simple, noetherian domain? This should give some clues for understanding $\operatorname{End}_{\mathscr{D}}(\mathscr{D} / L)$ when $L$ is a more general right ideal of $\mathscr{D}$.

\section{REFERENCES}

[AM] M. Atiyah and I. G. Macdonald, Commutative algebra, Addison-Wesley, London, 1969.

[BGG] J. N. Bernstein, I. M. Gelfand, and S. I. Gelfand, Differential operators on the cubic cone, Russian Math. Surveys 27 (1972), 169-174.

[Bj] J.-E. Bjork, Rings of differential operators, North-Holland Math. Library, Amsterdam, 1979.

[B] N. Bourbaki, Algèbre homologique, Hermann, Paris, 1984.

[C] J. R. Cannon, The one dimensional heat equation, Addison-Wesley, Menlo Park, Calif., 1984.

[DE] M. G. M. van Doorn and A. R. P. van den Essen, $\mathscr{D}_{n}$-modules with support on a curve, Publ. RIMS Kyoto Univ. 23 (1987), 937-953.

[G] A. Grothendieck, Eléments de géométrie algébrique IV, Inst. Hautes Études Sci. Publ. Math. 32 (1967).

[J] A. Joseph, The minimal orbit of a simple Lie algebra and the associated maximal ideal, Ann. École Norm. Sup. 9 (1976), 1-30.

[L] T. Levasseur, Sur la dimension de Krull de U(sl(3)), J. Algebra 102 (1986), 39-59.

[LS] T. Levasseur and J. T. Stafford, Rings of differential operators on classical rings of invariants, Mem. Amer. Math. Soc. 412 (1989).

[LSS] T. Levasseur, S. P. Smith, and J. T. Stafford, The minimal nilpotent orbit, the Joseph ideal and differential operators, J. Algebra 116 (1988), 480-501.

[Mal] B. Malgrange, Existence et approximation des solutions des équations aux derivées partielles et équations de convolution, Ann. Inst. Fourier (Grenoble) 6 (1955), 271-355.

[Mat] S. Matsuura, Factorization of differential operators and decomposition of solutions of homogeneous equations, Osaka J. Math. 15 (1963), 213-231.

[MR] J. C. McConnell and J. C. Robson, Noncommutative Noetherian rings, Wiley, London, 1988.

[P] P. Perkins, Commutative subalgebras of the ring of differential operators on a curve, Pacific J. Math. 139 (1989), 279-302.

[SS] S. P. Smith and J. T. Stafford, Differential operators on an affine curve, Proc. London Math. Soc. 56 (1988), 229-259.

[S1] S. P. Smith, Curves, differential operators and finite dimensional algebras, Séminaire d'Algèbre Paul Dubreil et Marie-Paul Malliavin, Lecture Notes in Math., vol. 1296, SpringerVerlag, 1986, pp. 158-176.

[St1] P. F. Stiller, Some applications of algebraic geometry to systems of partial differential equations and to approximation theory, Lefschetz Centennial Conf., Contemp. Math., vol. 58, Amer. Math. Soc., Providence, R. I., 1986, pp. 239-250.

[St2] Vector bundles on complex projective spaces and systems of pdes. I, Trans. Amer. Math. Soc. 298 (1986), 537-548. 
[T] F. Treves, Linear partial differential operators, Gordon and Breach, New York, 1970.

[W] D. V. Widder, The heat equation, Academic Press, New York, 1965.

Department of Mathematics, University of Washington, Seattle, Washington 98195 E-mail address: smith@math.washington.edu 